\title{
Novel Approaches for Detection Fluorescent-Labeled by Cellvizio Lab System on Hippocampal CA1 Region
}

\author{
Asma Ulhusna Shaimi ${ }^{1}$, Wan Raihana Wan Aasim², Hasmah Abdullah ${ }^{3}$, Wan Amir Nizam Wan Ahmad, \\ Tan Soo Choon ${ }^{4}$, Ang Chee Wei ${ }^{4}$, Zalina Ismail ${ }^{1, *}$ \\ ${ }^{1}$ BRAINetwork Centre for Neurocognitive Science, School of Health Sciences, Universiti Sains Malaysia, 16150 Kubang Kerian, Kelantan, Malaysia. \\ ${ }^{2}$ Malaysian Technology Development Corporation Sdn. Bhd., Level 8-9, Menara Yayasan Tun Razak, Malaysia. \\ ${ }^{3}$ School of Health Sciences, Universiti Sains Malaysia, 16150 Kubang Kerian, Kelantan, Malaysia. \\ ${ }^{4}$ Institute for Research in Molecular Medicine (INFORMM), Universiti Sains Malaysia, 11800 Pulau Pinang, Malaysia
}

Author correspondency*:

drzalina@gmail.com

\begin{abstract}
Neurosteroids have been identified in the 1981. Dehydroepiandrosterone sulphate (DHEAS) is one of the vital neurosteroids that de novo synthesized in the nervous system from cholesterol precursor (Baulieu \& Robel, 1998). The aim of the study is to develop a method for fluorescence labelling. Alexa Fluor 488 dye with DHEAS antibody can binds the DHEAS antibody in the rat brain monitored by Cellvizio Lab System. DHEAS antibody (IgG isotype antibodies) was fluorescently conjugated by an amine-reactive compound, Alexa Fluor 5SDP ester 488 dye. The resultant Alexa Fluor 488-conjugated antibodies were collected and analyzed by UV-Vis spectrophotometer instrument. The absorbance of the protein-dye conjugate at $280 \mathrm{~nm}$ and $494 \mathrm{~nm}$ were measured. Then, the degree of labeling (DOL) was calculated to achieve the desired results. Fluorescence labelling were carried out into the CA1 region of hippocampus Sprague-Dawley rat. We reported that the conjugation was successful. Optimal labeling depending on degree of labeling (DOL) needs some necessity to achieve and effective binding to the target neurosteroid, DHEAS. Cellvizio Lab system connected with Fiber Fluorescence Microscopy (FFM) probe is presented as a new approach in real-time imaging of DHEAS. In conclusion, we have developed a new method of DHEAS-Alexa Fluor fluorescence labelling to visualize and evaluate the changes of DHEAS fluorescence level in the rat hippocampus. This novel approach as a diagnostic tool and can be used to better understand the mechanisms and functions of DHEAS and other neurosteroids in future research.
\end{abstract}

Keywords: DHEAS; Alexa Fluor 488 dye; fluorescence labelling; Cellvizio Lab system

\section{INTRODUCTION}

Dehydroepiandrosterone sulphate (DHEAS) is a steroid which naturally synthesized in the brain. The steroids that are synthesized in the nervous system have been termed 'neurosteroids' (Baulieu, 1981). Baulieu \& Robel (1998), reported that concentrations of DHEAS are much higher in the brain than in plasma. The DHEAS can enhanced short term and long term memory performances in a variety of learning tasks.

Treatment aged animals with DHEAS enhance learning and memory in the T-maze footshock avoidance apparatus (Farr et al., 2004). Some studies have demonstrated that DHEAS enhancing memory performances in aged animals (Michael et al., 2001; Vallée et al., 2001). Behavior experiments that use the Morris water maze, Y maze, and radial arm water maze in animal models of learning and memory showed that DHEAS supplementation significantly affect behavioural performance (Bodensteiner et al., 2008). Another study in ovariectomized rats, i.c.v. implants by DHEAS may decrease escape latencies and distances to platform in the Morris water maze (Frye \& Sturgis, 1995)
In a study that assessed of learning and memory, fluorescence labelling was develop to conjugate between DHEAS monoclonal antibodies and Alexa Fluor 5-SDP ester 488 dye. Thus, a sulphodichlorophenyl ester (SDP) functional group is attached to a fluorophore core, Alexa Fluor 488 dye and bind specificity with monoclonal antibodies directly to form fluorophore-antibody conjugates. Moreover, SDP ester indicated hydrolytically stable form of the Alexa Fluor 488 dye for amine-conjugations (Molecular Probes, 2007). Vira and co-reseachers (2010) reported that the steric hindrance and the absence of additional reactive sites on the fluorophore may affect the conjugation reaction.

Conjugation between Alexa Fluor dye and antibodies were given exceptionally bright and most photostable (Panchuk-Voloshina et al., 1999). In fluorescence labelling, achieveing an optimal degree of labeling (DOL) and minimizing fluorescence quenching are the keys for conjugation (Berlier et al., 2003). Conjugation at high molar ratios of the fluorophore toward antibodies may cause DOLs become high. Therefore, it can be principal to the fluorescence quenching and assume to dye-dye interactions (Randolph \& Waggoner, 1997). The molar coefficient $\left(\varepsilon_{\mathrm{dye}}\right)$ of the Alexa Fluor 488 dye 
is 71,000 and indicated DOLs between ranges 4 to 9 . Further, at high DOLs, the Alexa Fluor 488 dye undergo more resistant to fluorescence quenching.

The stability to $\mathrm{pH}$ of Alexa Fluor dye was monitored by measuring their absorption spectra in buffers at $\mathrm{pH}$ 4-9. The most stable conjugates were performed at $\mathrm{pH}$ 8.3. For labelling, Alexa Fluor 488 dye can be retained the intensity in conjugation with antibodies. In principle, any fluorescent dye can be chosen to conjugate protein for live cell imaging, and many new dyes with improved photochemical properties have been developed (Panchuk-Voloshina et al. 1999). To complement a develop method of fluorescence labelling, Cellvizio Lab system connected with FFM probe will be used as an imaging approach to visualize and quantify the fluorescence intensity in vivo and real time imaging.

The Cellvizio Lab system provides to visualize deep brain through fluorescence imaging, high resolution, rapid, in real time image, with minimally invasive, and one animal at one time. Besides, stereotaxis apparatus as a platform to position the Fiber Fluorescence Microscopy (FFM) probe to the exact coordinate of target location (Visualsonics, 2009). The probe, an approach that designed for cannula implantation on anaesthetized animals, consisting of a diameter tip 0.30 $\mathrm{mm}$, lateral resolution $3.3 \mu \mathrm{m}$, optical sectioning $15 \mu \mathrm{m}$, and maximum field of view $600 \times 500 \mu \mathrm{m}$ (Vincent et al., 2006). Therefore, to understand how the brain activity of rats achieving in behavioral tasks, the Cellvizio Lab system with flexible FFM probe used to record in vivo imaging attached with DHEAS-Alexa Fluor fluorescence labelling at the region of interest.

In this article, we report the successful of DHEASAlexa Fluor 488 was expressed in the CA1 region of hippocampus rat brain. Here, our goal was to highlight a new method of DHEAS-Alexa Fluor 488 as a fluorescence labelling of DHEAS and expressed the signal of DHEAS by FFM connected with Cellvizio Lab system in visualizing real time imaged, hence as a new imaging approach based on fluorescence.

\section{MATERIALS AND METHODS}

\section{Antibody and reagents preparation}

DHEAS monoclonal antibodies $(3 \mathrm{mg} / \mathrm{mL})$ were bought from CalBioreagents. Alexa Fluor SDP Ester labeling dye (Thermo Scientific) was used to conjugate with antibodies. The corresponding fluorophore: protein (F:P) ratio, based on $\mathrm{A}_{280}$ and $\mathrm{A}_{494}$ absorption readings, was calculated according to the labeling instructions including the recommended correction factors for the absorbance of the dye at $280 \mathrm{~nm}$.

\section{Evaluation of dye delivery approaches in CA1 region of hippocampus}

All procedures were approved by the Animal Ethics Committee Universiti Sains Malaysia and followed the guidelines of the Animal Research and Service Centre. One group $(n=7)$ male Sprague-Dawley (SD) rats (250$300 \mathrm{~g}$ ) anaesthetised with isoflurane. SD rats were housed in groups of two per cage with twelve-hour light/dark cycle and food and water was provided $\mathrm{ad}$ libitum. All surgeries were performed under isoflurane anaesthesia. Anaesthesia was maintained by mask inhalation of isoflurane vaporized at concentrations of up $4 \%$ in the induction phase, at $2.5 \%$ during surgical procedures and at $1.0-1.5 \%$ during prolonged experimental observations. DHEAS, neurosteroid in the brain were imaged by using a beveled tip FFM probe through a hole in the skull. The probe was positioned by using the holder of motorized stereotaxis device. One microliter of DHEAS-Alexa Fluor 488 dye was carried out into the CA1 region using $10 \mu \mathrm{L}$ Hamilton syringe, set to eject $1.00 \mu \mathrm{L}$ over about $10 \mathrm{~min}$. Injection coordinates were (in $\mathrm{mm}$ ): for the CA1 hippocampus, AP: $-3.00 \mathrm{~mm}$, ML: $-1.7 \mathrm{~mm}$ and DV: $3.00 \mathrm{~mm}$. Optical recording started $1 \mathrm{~h}$ later.

\section{Preparation of Alexa Fluor 5-SDP ester 488 dye labelled monoclonal antibodies}

Dehydroepiandrosterone sulphate (DHEAS) was fluorescently conjugated to an amine-reactive compound, Alexa Fluor 5-SDP ester 488 dye (Invitrogen). DHEAS solution $(3.0 \mathrm{mg} / \mathrm{mL})$ was exchanged to sodium bicarbonate by dialysis with against $0.1 \mathrm{M}$ sodium bicarbonate solution ( $\mathrm{pH}$ 8-9) for 2 hours at room temperature. Then, Alexa Fluor 488 dye were prepared in DMF solution at concentration 10 $\mathrm{mg} / \mathrm{mL}$. Therefore, $1.2 \mu \mathrm{L}$ reactive dye was added into the $100 \mu \mathrm{g}$ DHEAS solution and incubate the solution for 1 hour at room temperature. The resultant solution was transferred into the membrane dialysis tube and dialyzed against PBS (pH 7.4) an overnight in $4^{\circ} \mathrm{C}$ with continuous stirring. The resultant DHEAS-Alexa Fluor 488 was collected and sterilized via $200 \mathrm{~nm}$ polymer membrane filter and was stored at $4{ }^{\circ} \mathrm{C}$ and $-20^{\circ} \mathrm{C}$ for long storage.

The concentration of DHEAS antibodies and Alexa Fluor dye were calculated from the absorbance at 280 and $488 \mathrm{~nm}$, measured using an ultraviolet (UV-Vis) spectrophotometer, using the following formula:

The concentration of protein in the sample:

Protein concentration $(\mathrm{M})=$

$$
\frac{\left[\mathrm{A}_{280}-\left(\mathrm{A}_{\text {dye }} \times \mathrm{CF}_{280}\right)\right] \times \text { dilution factor }}{203,000}
$$


The degree of labeling:

Moles dye per mole protein $=$

$\mathrm{A}_{\text {dye }} \mathrm{x}$ dilution factor

$\overline{\varepsilon_{\text {dye }} \mathrm{x} \text { protein concentration (M) }}$

(Molecular Probe, 2011)

Real time imaging by Fiber Fluorescence Microscopy (FFM) probe connected with Cellvizio Lab system

After labelling, rats were anaesthetised and positioned on a stereotaxis apparatus. The FFM probe with a holder was placed into the mount of the stereotaxis device. The probe was fixed into position on top of the cannula implantation and set the coordinate as bregma point $(0,0,0)$. Next, the FFM probe connected with Cellvizio Lab system (Le Goualher et al., 2004; Davenne et al., 2005) and a frame rate was at $12 \mathrm{~Hz}$. Excitation of cells at $488 \mathrm{~nm}$ laser light and emission was measured at 494 $\mathrm{nm}$. The Cellvizio Lab system have a single-pixel avalanche photodiode detector (APD) for resolution and sensitivity, respectively.

\section{RESULTS}

\section{Development of DHEAS-Alexa Fluor 488 fluorescence labelling}

We conjugated DHEAS antibody with reactive dye, excitation and emission wavelength at $488 \mathrm{~nm}$ and 494 $\mathrm{nm}$, respectively. For this purpose, we determined by measuring the optimal degree of labeling with different concentration of protein, wherein the buffer $\mathrm{pH}$ was constant. Table 1 show the absorbance of DHEAS antibody and reactive dye based on $\mathrm{A}_{280}$ and $\mathrm{A}_{494}$ absorption reading, the concentration of protein, dilution factor, degree of labeling, and molar coefficient of specific dye and correction factor for the fluorophore contribution as constant variables. Based the result, the absorption reading for antibody and dye increase, the degree of labeling of fluorescence intensity was increased. Therefore, according to obtained result, concentration of antibody, $0.91 \mathrm{M}$ and degree of labeling, 4.63 was chosen for this present study as a new method.

Table 1. The protein concentration and degree of labeling in different protocols.

\begin{tabular}{|c|c|c|c|c|c|c|c|c|c|}
\hline 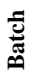 & $\mathbf{A}_{280}$ & $\mathbf{A}_{494}$ & {$[\mathbf{P}]$} & ○̊ & dl & $\varepsilon_{\text {dye }}$ & $\mathrm{CF}_{280}$ & $\lambda_{\max }$ & Em \\
\hline 1 & 0.82836 & 0.57950 & 0.57 & 2.17 & 1 & 71,000 & 0.11 & 494 & 519 \\
\hline 1 & 1.64540 & 0.92632 & 1.14 & 1.70 & 1 & \multirow{3}{*}{\multicolumn{4}{|c|}{$*^{*}$ constant variables }} \\
\hline 2 & 1.18320 & 1.44100 & 1.51 & 4.02 & 2 & & & & \\
\hline 3 & 1.45050 & 1.99340 & 0.91 & 4.63 & 1 & & & & \\
\hline
\end{tabular}

* $\mathrm{A}_{280}$ and $\mathrm{A}_{494}$ is an absorption reading by UV-Vis spectroscopy.

* $[\mathrm{P}]$ is protein concentration in $\mathrm{mg} / \mathrm{mL}$.

* DOL is degree of labeling.

$* \mathrm{dl}$ is dilution factor to modify the calculation.

$* \varepsilon_{\text {dye }}$ is molar extinction coefficient at $\lambda \max$ in $\mathrm{cm}^{-1} \mathrm{M}^{-1}$

$* \mathrm{CF}_{280}$ is correction factor for absorption readings at $280 \mathrm{~nm}$.

$* \lambda_{\max }$ and Em are the fluorescence absorbance and emission maxima, in $\mathrm{nm}$ conjugated to an IgG antibody.
Conjugation of DHEAS-Alexa Fluor 488 as fluorescence labelling

A standardized protocol was implemented in this experiment, and yield less than 4 of DOLs of conjugation DHEAS-Alexa Fluor 488. The experimental workflow has made some modification and introduced two principals in a new method. First, the time incubation need to be longer for conjugation. The conjugates were incubated overnight to increase chemically binding of the DHEAS-Alexa Fluor conjugation. To achieve effective labelling, Marks et al. (2004) reported that it required the incubation of $1 \mu \mathrm{M}$ 5'-Fluorescein-SLF' for 16 hours. The second modification was increased the volume of the dye from stock solution against antibody to enhance the expression signal of DHEAS-Alexa Fluor fluorescence intensity. The standard protocol of dye was $1.2 \mu \mathrm{L}$ dye in $100 \mu \mathrm{g}$ antibody $(10 \mathrm{mg} / \mathrm{mL})$. Therefore, in this study the method was modified, $2.4 \mu \mathrm{L}$ dye in $100 \mu \mathrm{g}$ antibody. The UV spectra for batch 2 and 3 has shown in figure 1.
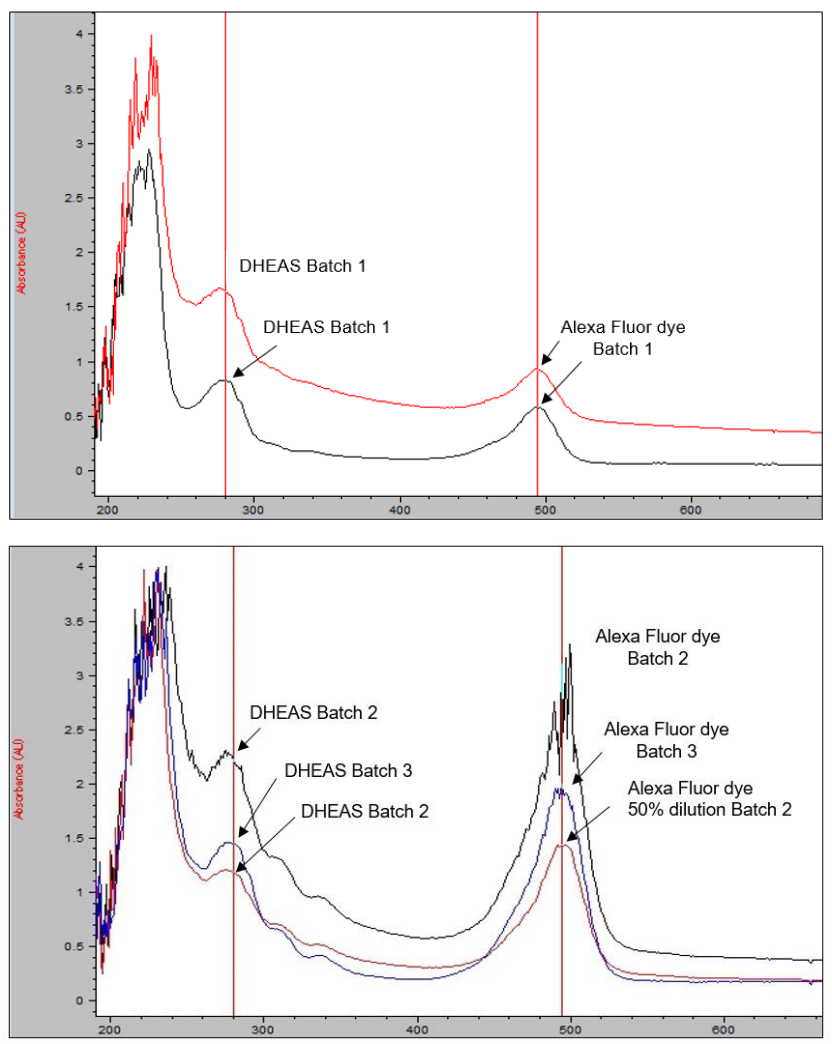

Figure 1. The UV spectra showing the absorption DHEAS antibody and Alexa Fluor dye for batch 1, batch 2 and batch 3 . The absorption peaks at $288 \mathrm{~nm}$ and $494 \mathrm{~nm}$ correspond to the absorption for antibody and excitation of Alexa Fluor 488 dye, respectively.

\section{Expression of DHEAS-Alexa Fluor fluorescence labelling by FFM Imaging}

FFM probe attached to Cellvizio Lab system has been used to monitor DHEAS fluorescence levels in the hippocampal CA1 region. The characteristics of 
microprobe S300B was shown in Table 2. SpragueDawley rat was positioned and placement on the motorized stereotaxis apparatus, then DHEAS-Alexa Fluor 488 fluorescence labelling was carried out into the CA1 region of hippocampus of anaesthetized animals. One hour after labelling, FFM imaging was visualized and imaged the signal of DHEAS fluorescence expression. The changes of DHEAS fluorescence expression was quantified and recorded the images at region of interest (ROI)s in real time imaged and minimally invasiveness.

Table 2. Properties of the microprobe S-300B used in this study.

\begin{tabular}{ll}
\hline Probe & S-300B \\
\hline Diameter $(\mathrm{mm})$ & 0.30 \\
Maximum field of view $(\mu \mathrm{m} \times \mu \mathrm{m})$ & $600 \times 500$ \\
Axial resolution $(\mu \mathrm{m})$ & 15 \\
Lateral resolution $(\mu \mathrm{m})$ & 3.3 \\
\hline Values provided by Cellvizio Lab system, Mauna Kea Technologies, \\
France
\end{tabular}
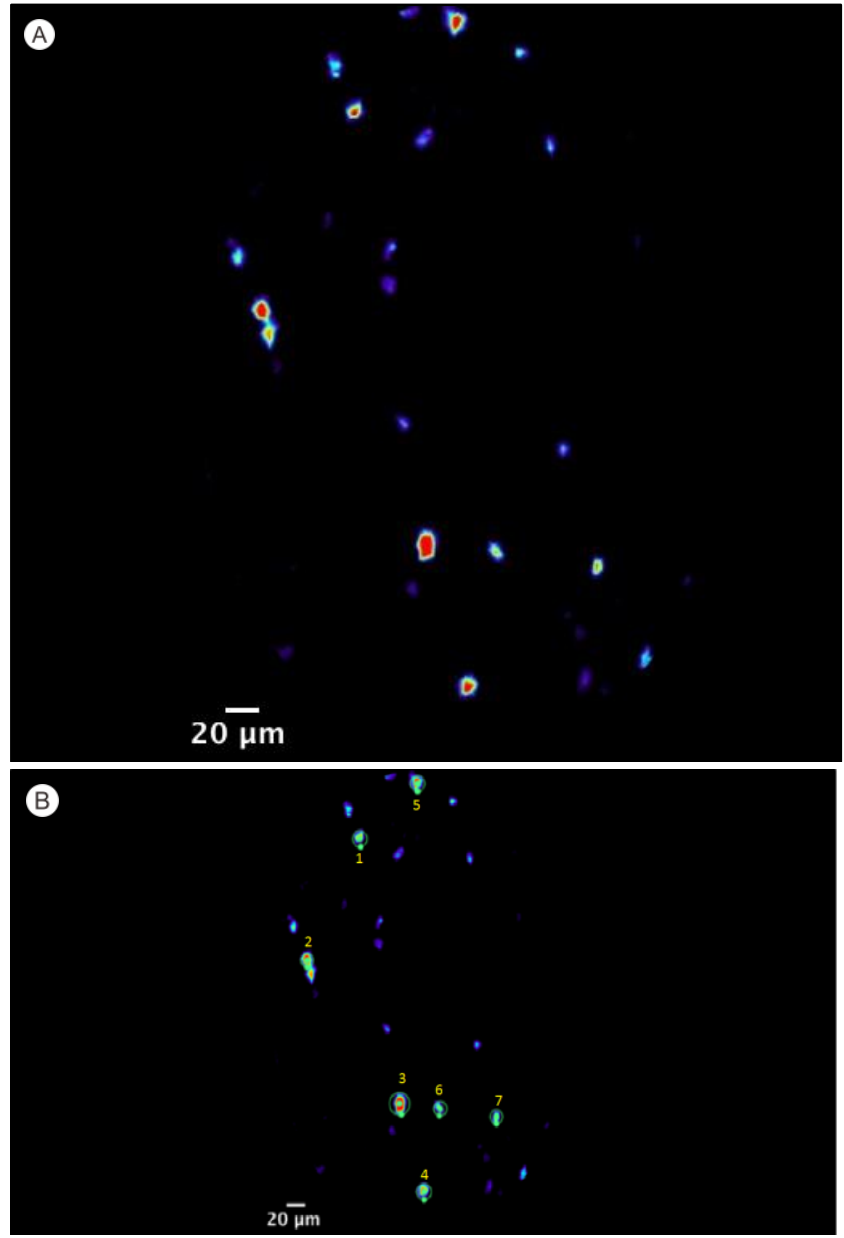

Figure 2. The signal of DHEAS fluorescence expression. (A) The images visualized indicated the DHEAS fluorescence was expressed the signal in hippocampal CA1 region. (B) The region of interest (ROI) of DHEAS fluorescence signal were selected.

\section{DISCUSSION}

The excitation and emission spectra of Alexa Fluor 488 dye are illustrated in Figure 3. Figure 1 in this present study demonstrated the UV-Vis spectra of Alexa Fluor 5-SDP ester 488 dye conjugated with DHEAS antibodies. The UV-Vis spectrophotometer is a useful instrument in most laboratories (Shahal et al., 2016). The aim of this study was to develop method of DHEAS-Alexa Fluor fluorescence labelling and evaluate the successful conjugation to be used as a tagging for DHEAS in the CA1 region of hippocampus by imaging approach, FFM connected with Cellvizio Lab system. This finding indicated that the antibody conjugation was effectively and efficiently to be used as fluorescence labelling to image and quantify DHEAS in the hippocampus rat brain by FFM imaging.

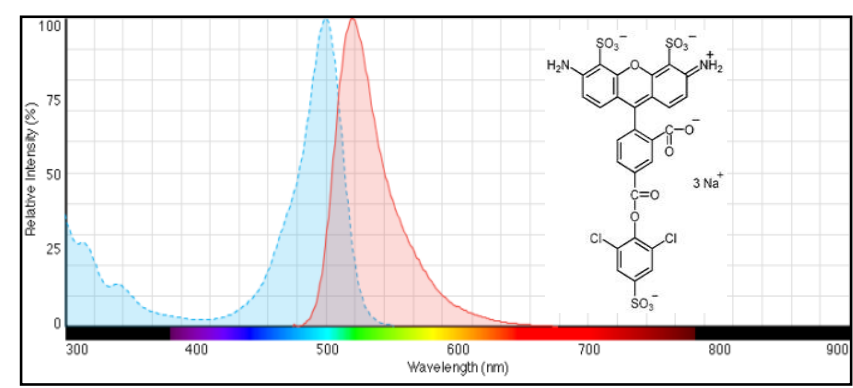

Figure 3. The illustration of excitation and emission spectra of Alexa Fluor 488 dye.

Coons and co-researchers (1950) showed that antibody conjugation widely uses nowadays in the biological sciences and medicine. In conjunction, labelling method is a direct technique which is the antibodies chemically conjugated to a fluorescent dye. The result of this study indicated that conjugation between Alexa Fluor 488 dye and DHEAS antibodies was succeed. The result of the present study also suggest that based on calculation degree of labeling, the F:P ratio the conjugation should be in range 4 to 9 (Table 1 ). This finding indicated that fluorescence labelling can be effective used and given brighter expression in animal studies (Hayashi-Takanaka et al., 2014; Vira et al., 2010).

In order to obtain better labelling, the parameters that can affected the fluorescence should be considered. Firstly, the antibodies and reactive dye should be in the same buffer. In this case, $0.1 \mathrm{M}$ sodium bicarbonate was used to change the buffer of antibodies. It is necessary to achieve strongly binding in the covalent bond of conjugation. Furthermore, longer incubation time required for fluorescence labelling protocols to determine the optimal DOL of DHEAS-Alexa Fluor 488 conjugates. This suggested that the conjugation leaves for an overnight or at least 16 hours to increase the fluorescence intensity. The absorption data from UV-Vis spectra was increased corresponding to the time of incubation. Additionally, we modified the approach of 
the dye from standard labeling protocols to increase the efficiency of labeling. Initial experiment was conducted with preparation dye in standard protocol $(1.2 \mu \mathrm{L}$ dye in $100 \mu \mathrm{g}$ antibody). We altered the dye which is increase the volume of dye from stock solution into twice time, $2.4 \mu \mathrm{L}$ dye in $100 \mu \mathrm{g}$ antibody. The results in this present study demonstrated that increased the DOL of conjugation.

There are have several requirements were highlighted in order to achieve the better labelling. This could be due these findings accordance with the previous studies and clearly demonstrated of brightness, photostability, photobleaching, binding affinity, size of fluorophore, fluorescent lifetime and molar coefficient (Hayashi-Takanaka et al., 2014; Shaner et al., 2005; Toseland, 2013). Additionally, better expression of fluorescent labelling influence by $\mathrm{pH}$ sensitivity (Shaner et al., 2005). Thus, $\mathrm{pH}$ conditions performed as an indicator of fluorescent lifetime in conjugation of imaging living cells (Lin et al., 2003).

The FFM probe was inserted into the brain to recorded and quantified the changes of DHEAS fluorescence intensity level in the CA1 region of hippocampus. Surprisingly, this present study is the first report to evaluate the expression of DHEAS fluorescence in the target area, CA1 area of hippocampus by FFM connected with Cellvizio Lab system. According to Vincent et al. (2006) demonstrated that Cellvizio Lab system allowed researchers to visualize calcium signals in deep brain structures. These results are consistent with FFM connected with Cellvizio Lab system that allowed the direct recording in a short time frame, same animals can be used repeatedly, avoiding shrinkage of cells and tissues, and flexible probes (Frykman et al. 1988). Thus, FFM connected with Cellvizio Lab system was essential for in vivo imaging associated with behavioral task in order to time saving and does not require sample preparation or staining to prove the presence of fluorescence labelling.

In this present study showed the signal of DHEAS fluorescence expressed at the region of interest (Figure 2). This finding indicate that the signal of expression DHEAS fluorescence can be used as a potential in vivo imaging to evaluate and quantify the changes of DHEAS level in the rat brain due to physiology, pathology and pharmacology process (Vincent et al., 2006; Visualsonics, 2009). Accordingly, the results in this study presumed that the changes of DHEAS fluorescence expression associated with cognition. We have developed new method of fluorescence labelling in conjunction with fluorescence imaging approach, FFM connected with Cellvizio Lab system that precise and accurate visualization of fluorescence signals in living brains as well as non-invasive method for cellular level imaging in vivo (Davenne et al., 2005; Vincent et al., 2006; Makhlouf et al., 2008).

\section{CONCLUSION}

In conclusion, DHEAS-Alexa Fluor 488 fluorescence labelling was developed to monitor and evaluate the changes of DHEAS fluorescence level that expressed in the CA1 region of hippocampus. Beyond imaging, the DHEAS-Alexa Fluor fluorescence labelling is one step approach to monitor dynamic behavioral task and better understand the mechanisms of DHEAS. Moreover, this approach can be used to discover the beneficial functions of DHEAS and other neurosteroids in the hippocampus at preclinical level to encourage in future research.

\section{ACKNOWLEDGEMENT}

This research was supported by the FRGS grant (203/PPSK/6171153) and Universiti Sains Malaysia.

\section{REFERENCES}

Baulieu, E. E. 1981. Steroid Hormone Regulation of the Brain, eds. Fuxe, K. \& Gustafsson, J. A. (Pergamon, Oxford), 3-14.

Baulieu, E. E. \& Robel, P. 1998. Dehydroepiandrosterone (DHEA) and dehydroepiandrosterone sulfate (DHEAS) as neuroactive neurosteroids. Proc. Natl. Acad. Sci., 95:40894091.

Berlier, J. E., Anca, R., Gayle, B., Jolene, B., Diane, R. G., Brian, J. F., William, G. T., Yue, S., Liu, J., Cheung, C., Chang, W., James, D. H., Joseph, M. B., Rosaria, P. H. \& Richard, P. H. 2003. Quantitative Comparison of Long-wavelength Alexa Fluor Dyes to Cy Dyes: Fluorescence of the Dyes and Their Bioconjugates. The Journal of Histochemistry \& Cytochemistry, 51(12): 1699-1712.

Bodensteiner, K. J., Stone, I. J., \& Ghiraldi, L. L. 2008. Effects of Dehydroepiandrosterone Sulfate and Progesterone on Spatial Learning and Memory in Young and Aged Mice. The Journal of General Psychology, 135(3): 271-286.

Coons, A. H., \& Kaplan, M. H. 1950. Localization of antigen in tissue cells; improvements in a method for the detection of antigen by means of fluorescent antibody. The Journal of Experimental Medicine, 91(1): 1-13.

Davenne, M., Custody, C., Charneuau, P. \& Lledo, P. M. 2005. In vivo Imaging of Migrating Neurons in the Mammalian Forebrain. Chemical Senses, 30:115-116.

Farr, A. S., William, A. B., Kayoko, U. Gaskin, F. S. \& John, E. M. 2004. DHEAS improves learning and memory in aged SAMP8 mice but not in diabetic mice. Life Sciences, 75: 2775-2785.

Frye, C. A. \& Sturgis, J. D. 1995. Neurosteroids affect spatial/reference, working, and long-term memory of female rats. Neurobiology of Learning and Memory, 64: 83-96.

Frykman, G. K., McMillan, P. J. \& Yegge, S. A. 1988. A review of experimental methods measuring peripheral nerve regeneration in animals. OrthopClin N Am., 19: 209-219.

Hayashi-Takanaka, Y., Stasevich, T.J., Kurumizaka, H., Nozaki, N. \& kimura, H. 2014. Evaluation of chemical fluorescent dyes as protein conjugation partner for live cell imaging. PLOS ONE, 9(9): 1-11. 
Le Goualher, G., Perchant, A., Genet, M., Cavé, C., Viellerobe, B., Berier, F., Abrat, B. \& Ayache, N. 2004. Towards Optical Biopsies with an Integrated Fibered Confocal Fluorescence Microscope. 3217:761-768.

Lin, H., Herman, P., \& Lakowicz, J. R. 2003. Fluorescence Lifetime-Resolved $\mathrm{pH}$ Imaging of Living Cells. Cytometry Part A, 52A: 77-89.

Makhlouf, H., Gmitro, A. F., Tanbakuchi, A. A., Udovich, J. A. \& Rouse, A. R. 2008. Multispectral confocal microendoscope for in vivo and in situ imaging. J. Biomed Opt., 13(4): 1-21.

Marks, K. M., Braun, P. D. \& Nolan, G. P. 2004. A general approach for chemical labeling and rapid, spatially controlled protein inactivation. PNAS, 27(101): 9982-9987.

Michael, M., Michelle, U., Daniel, B. \& Charles, L. 2001. Memory-enhancing effects of DHEAS in aged mice on a winshift water escape task. Physiology \& Behavior, 72: 521-525.

Molecular Probes. 2007. Technical Tip: Greater Control and More Reaction Time with SDP Ester. Invitrogen.

Molecular Probes. 2011. Alexa Fluor 488 Monoclonal Antibody Labeling Kit. Invitrogen.

Panchuk-Voloshina, N., Rosaria, P. H., Janell, B. S., Mahesh, K. B., Paul, J. M., Fei, M., Leung, W. \& Richard, P. H. 1999. Alexa Dyes, a Series of New Fluorescent Dyes that Yield Exceptionally Bright, Photostable Conjugates. The Journal of Histochemistry \& Cytochemistry, 47(9):1179-1188.

Randolph, J. B. \& Waggoner, A. S. 1997. Stability, specificity and fluorescence brightness of multiple-labeled fluorescent DNA probes. Nucleic Acids Res., 25: 2923-2929.
Shahal, T., Green, O., Hananel, U., Michaeli, Y., Shabat, D., \& Ebenstein, Y. 2016. Simple and cost-effective fluorescent labeling of 5-hydroxymethylcytosine. Methods and Applications in Fluorescence, 4: 1-7.

Shaner, N. C., Steinbach, P. A. \& Tsien, R. Y. 2005. A guide to choosing fluorescent proteins. Nature Methods, 2(12): 905909.

Toseland, C. P. 2013. Fluorescent Labeling and modification of proteins. J Chem Biol, 6: 85-95.

Vallée, M., Mayo, W. \& Moal, M. L. 2001. Role of pregnenolone, dehydroepiandrosterone and their sulfate esters on learning and memory in cognitive aging. Brain Research Reviews, 37: 301-312.

Vincent, P., Maskos, U., Charvet, I., Bourgeais, L., Stoppini, L., Leresche, N., Changeux, J., Lambert, R., Meda, P. \&Paupardin-Tritsch, D. 2006. Live imaging of neural structure and function by fibred fluorescence microscopy. European Molecular Biology Organization Reports, 7(11):1154-1161.

Vira, S., Elena, M., Glen, H. \& Paul, S. B. 2010. Fluorescent labeled antibodies-balancing functionality and degree of labeling. Anal Biochem., 402(2): 146-150.

Visualsonics. 2009. Application Brief: Cellvizio Lab Fibered Fluorescence Microscopy and Microdialysis Research. 